\title{
MAXIMAL IDEALS AND THE STRUCTURE OF CONTRACTIBLE AND AMENABLE BANACH ALGEBRAS
}

\author{
YONG ZHANG
}

\begin{abstract}
Properties of minimal idempotents in contractible and reflexive amenable Banach algebras are exploited to prove that such a kind of Banach algebra is finite dimensional if each maximal ideal is contained in a maximal left or a maximal right ideal that is complemented as a Banach subspace. This result covers several known results on this subject.
\end{abstract}

\section{InTRODUCtion}

Suppose that $\mathfrak{A}$ is a Banach algebra over the complex field $\mathbb{C}$ and $X$ is a Banach $\mathfrak{A}$-bimodule. A derivation from $\mathfrak{A}$ into $X$ is a linear operator $D: \mathfrak{A} \rightarrow X$ which satisfies $D(a b)=a \cdot D(b)+D(a) \cdot b, a, b \in \mathfrak{A}$. For any $x \in X$, the mapping $\delta_{x}: \mathfrak{A} \rightarrow X$ given by $\delta_{x}(a)=a x-x a, a \in \mathfrak{A}$, is a continuous derivation, called an inner derivation. A Banach algebra $\mathfrak{A}$ is said to be contractible if for every Banach $\mathfrak{A}$-bimodule $X$ each continuous derivation from $\mathfrak{A}$ into $X$ is inner. If $X$ is a Banach $\mathfrak{A}$-bimodule, then $X^{*}$, the conjugate space of $X$, is naturally a Banach $\mathfrak{A}$-bimodule with the module actions defined by

$$
\langle x, a f\rangle=\langle x a, f\rangle, \quad\langle x, f a\rangle=\langle a x, f\rangle, \quad\left(a \in \mathfrak{A}, f \in X^{*}, x \in X\right),
$$

where $\langle x, f\rangle$ denotes the evaluation of $f$ at $x$. A Banach algebra $\mathfrak{A}$ is said to be amenable if for every Banach $\mathfrak{A}$-bimodule $X$ each continuous derivation from $\mathfrak{A}$ into the dual module $X^{*}$ is inner. It is a basic fact that a contractible Banach algebra has an identity and an amenable Banach algebra has a bounded approximate identity. We call a Banach algebra a reflexive Banach algebra if the underlying space is reflexive as a Banach space. Since a reflexive Banach algebra is weak* complemented, a reflexive amenable Banach algebra has an identity. In this paper we simply denote the identity element in a Banach algebra by 1 .

The structure of contractible and reflexive amenable Banach algebras has been studied by many authors. It is a simple fact that any finite dimensional semi-simple Banach

Received 16th November, 1999

The author wishes to thank Professor F Ghahramani for bringing the problem to him and for many illuminating conversations.

Copyright Clearance Centre, Inc. Serial-fee code: 0004-9727/00 \$A2.00+0.00. 
algebra is contractible and, of course, is (reflexive) amenable (see [5, I.3.68 and VII.1.74]). The converse. is known to be true for some special cases: A contractible Banach algebra is finite dimensional if it has the bounded (compact) approximation property [9] or if it is commutative [2]. As to reflexive amenable Banach algebras, it has been conjectured that they should also be finite dimensional. Gale, Ransford and White proved in [3] that this is true if irreducible representations of $\mathfrak{A}$ are finite dimensional. This result was improved by Johnson in [6], where he showed that this is the case if each maximal left ideal of $\mathfrak{A}$ is complemented as a Banach subspace. Later, Ghahramani, Loy and Willis showed in [4] that the conjecture is true if the underlying space of $\mathfrak{A}$ is a Hilbert space. Although this result is covered by Johnson's preceding result, the method is different and will be exploited in this paper. Recently, Runde [8] obtained some new results on this problem. One of his main results is that if each maximal ideal of $\mathfrak{A}$ is of finite codimension then the conjecture is true. In this paper we shall give a theorem which improves both Johnson's and Runde's results.

\section{Complemented left ideals}

Suppose that $\mathfrak{A}$ is a Banach algebra and $E \subset \mathfrak{A}$. The left annihilator and the right annihilator of $E$ are, respectively, the following sets

$$
\operatorname{lan}(E)=\{a \in \mathfrak{A} ; a E=\{0\}\}, \quad \operatorname{ran}(E)=\{a \in \mathfrak{A} ; E a=\{0\}\} .
$$

For an element $a \in \mathfrak{A}, \operatorname{lan}(\{a\})$ and $\operatorname{ran}(\{a\})$ will be simply denoted by $\operatorname{lan}(a)$ and $\operatorname{ran}(a)$ respectively. The following lemma is trivial.

LEMMA 1. Suppose that $\mathfrak{A}$ is a Banach algebra having an identity 1 . Then for any $a \in \mathfrak{A}$,

(i) $\operatorname{ran}(\mathfrak{A}(1-a))=\operatorname{ran}(1-a)=\{x \in \mathfrak{A} ; a x=x\}$,

(ii) $\operatorname{lan}((1-a) \mathfrak{A})=\operatorname{lan}(1-a)=\{x \in \mathfrak{A} ; x a=x\}$.

Recall that a non-zero element $e$ of $\mathfrak{A}$ is a minimal idempotent if $e$ is an idempotent (that is, $e^{2}=e$ ) and $e \mathfrak{A l}$ is a division algebra.

LEMMA 2. Suppose that $\mathfrak{A}$ is a contractible or a reflexive amenable Banach algebra. Let $L$ be a closed proper left (right) ideal of $\mathfrak{A}$. If $L$ is complemented in $\mathfrak{A}$, then $\operatorname{ran}(L)$ (respectively, $\operatorname{lan}(L)$ ) contains an idempotent $e$ such that the following hold.

(i) $\operatorname{ran}(L)=e \mathfrak{A}$ (respectively, $\operatorname{lan}(L)=\mathfrak{A} e$ );

(ii) $L=\mathfrak{A}(1-e)$ (respectively, $L=(1-e) \mathfrak{A})$;

(iii) If in addition, $L$ is a maximal left (respectively, right) ideal, then $e$ is a minimal idempotent and $\mathfrak{A} e$ (respectively, $e \mathfrak{A}$ ) is a minimal left (respectively, right) ideal. 
Proof: We prove the case when $L$ is a left ideal. To prove (i) and (ii) we can assume $L \neq\{0\}$, for otherwise $e=1$ satisfies the requirements. First we show that $L$ contains a right identity $\mu$. Then it is clear that $\mu$ is an idempotent and $L=\mathfrak{A} \mu$.

If $\mathfrak{A}$ is contractible, we consider the following exact short sequence of left $\mathfrak{A}$-modules:

$$
\sum: \quad 0 \longrightarrow L \stackrel{\mathfrak{s}}{\longrightarrow} \mathfrak{A} \stackrel{\mathfrak{q}}{\longrightarrow} \mathfrak{A} / L \longrightarrow 0,
$$

where $\imath$ is the inclusion mapping and $q$ is the quotient mapping. Since $L$ is complemented, $\sum$ is admissible. From [2, Theorem 6.1] $\sum$ is a splitting sequence, that is, there is a left A-module morphism $\delta: \mathfrak{A} \rightarrow L$, such that $\delta \circ \imath=I_{L}$, the identity operator on $L$. Then $\mu=\delta(1)$ is obviously a right identity of $L$.

If $\mathfrak{A}$ is reflexive and amenable, then according to [2, Theorem 3.7], $L$ contains a right bounded approximate identity, say $\left(l_{\alpha}\right)$. Since $\mathfrak{A}$ is reflexive, as a closed subspace of $\mathfrak{A}$, $L$ is also reflexive. Then a weak* cluster point $\mu$ of $\left(l_{\alpha}\right)$ in $L$ is a right identity of $L$.

Now let $e=1-\mu$. Then $e \in \operatorname{ran}(L), e \neq 0$ and $e$ is also an idempotent. Since $L=\mathfrak{A} \mu=\mathfrak{A}(1-e)$, from Lemma $1, \operatorname{ran}(L)=\{x \in \mathfrak{A} ; e x=x\}=e \mathfrak{A}$. This proves the first two statements of this lemma.

Now suppose that $L$ is a maximal left ideal. Then, since $\mathfrak{A}$ is the direct sum of $\mathfrak{A}(1-e)$ and $\mathfrak{A} e$, we have that $\mathfrak{A} e$ is a minimal left ideal of $\mathfrak{A}$. By [1, Lemma 30.2], $e$ is a minimal idempotent. This completes the proof.

In the following we use $\operatorname{rad}(\mathfrak{A})$ to denote the radical of $\mathfrak{A}$.

LEMMA 3. Suppose that $\mathfrak{A}$ is a contractible or a reflexive amenable Banach algebra. If $\mathfrak{A} / \operatorname{rad}(\mathfrak{A})$ is finite dimensional, then so is $\mathfrak{A}$ and $\mathfrak{A}$ is semi-simple.

Proof: If $\mathfrak{A} / \operatorname{rad}(\mathfrak{A})$ is finite dimensional, then $\operatorname{rad}(\mathfrak{A})$ is a complemented closed ideal of $\mathfrak{A}$. From Lemma $2 \operatorname{rad}(\mathfrak{A})$ contains an idempotent which is non-zero if $\operatorname{rad}(\mathfrak{A}) \neq$ $\{0\}$. But $\operatorname{rad}(\mathfrak{A})$ can never have a non-zero idempotent. So $\operatorname{rad}(\mathfrak{A})=\{0\}$.

It is known that if a Banach algebra is contractible or amenable, then its image under a continuous algebraic homomorphism is also contractible or amenable (see [5, Proposition VII.1.71] and [7, Proposition 5.3]). This leads to part of the following lemma.

LEmma 4. Suppose that $\mathfrak{A}$ is a Banach algebra. Then the following statements hold.

(i) If $\mathfrak{A}$ is contractible, or reflexive and amenable, then so is $\mathfrak{A} / \operatorname{rad}(\mathfrak{A})$;

(ii) An ideal $M \subset \mathfrak{A} / \operatorname{rad}(\mathfrak{A})$ is a maximal ideal if and only if $q^{-1}(M)$ is a maximal ideal in $\mathfrak{A}$, where $q: \mathfrak{A} \rightarrow \mathfrak{A} / \operatorname{rad}(\mathfrak{A})$ is the quotient mapping;

(iii) If $L$ is a maximal left (right) ideal of $\mathfrak{A}$ containing $\operatorname{rad}(\mathfrak{A})$, then $q(L)$ is a maximal left (respectively, right) ideal of $\mathfrak{A} / \operatorname{rad}(\mathfrak{A})$. If $L$ is complemented in $\mathfrak{A}$, then $q(L)$ is complemented in $\mathfrak{A} / \operatorname{rad}(\mathfrak{A})$.

Proof: The first statement is clear. Checking of the second one is also a routine. Suppose that $L$ is a left (right) ideal of $\mathfrak{A}$ and $\operatorname{rad}(\mathfrak{A}) \subset L$. Then it is easily verified that 
$L=q^{-1}(q(L))$. So $q(L)$ is maximal in $\mathfrak{A} / \operatorname{rad}(\mathfrak{A})$ whenever $L$ is maximal in $\mathfrak{A}$. If $L$ is complemented in $\mathfrak{A}$, then there is a closed complement, say $J$, of $L$ in $\mathfrak{A}$. The image $q(J)$ is also closed since $q$ is open, and

$$
q(L)+q(J)=\mathfrak{A} / \operatorname{rad}(\mathfrak{A}) .
$$

If $m \in q(L) \cap q(J)$, then for some $j \in J$ and $l \in L, m=q(j)=q(l)$. Hence $j-l \in \operatorname{rad}(\mathfrak{A}) \subset L$. So $j \in L$. This shows that $j=0$ and hence $m=0$. Therefore $q(J)$ is a complement of $q(L)$ in $\mathfrak{A} / \operatorname{rad}(\mathfrak{A})$. Thus $q(L)$ is complemented in $\mathfrak{A} / \operatorname{rad}(\mathfrak{A})$.

\section{Main Results}

THeOREM 5. Suppose that $\mathfrak{A}$ is a contractible or a reflexive amenable Banach algebra. If each maximal ideal of $\mathfrak{A}$ is contained in either a maximal left ideal or a maximal right ideal of $\mathfrak{A}$ which is complemented in $\mathfrak{A}$, then $\mathfrak{A}$ is finite dimensional.

Proof: From Lemmas 3 and 4 we can assume that $\mathfrak{A}$ is semi- simple. We can also assume that $\mathfrak{A}$ is not a division algebra. Then $\mathfrak{A}$ contains at least one maximal ideal and hence has at least one maximal left or maximal right ideal which is complemented in $\mathfrak{A}$. By Lemma 2, $\mathfrak{A}$ has at least one minimal idempotent. Let $E$ be the set all minimal idempotents of $\mathfrak{A}$, and let $J$ be the ideal generated by $E$ (the socle of $\mathfrak{A}$ ). We prove $J=\mathfrak{A}$.

If $J \neq \mathfrak{A}$, then there is a maximal ideal $M$ containing $J$, since $\mathfrak{A}$ has an identity. Then, by assumption, there is either a maximal left ideal or a maximal right ideal of $\mathfrak{A}$ which contains $M$ and which is complemented in $\mathfrak{A}$. Assume the former is true and the corresponding left ideal is $L$. Then from Lemma $2, \operatorname{ran}(L) \neq\{0\}$ and contains a minimal idempotent $e$ such that $L=\mathfrak{A}(1-e)$. So we would have $E e=\{0\}$ and $e \in E$. This implies that $e=e^{2}=0$, a contradiction.

Therefore $J=\mathfrak{A}$. Then the identity 1 of $\mathfrak{A}$ can be represented as

$$
1=\sum_{i=1}^{n} a_{i} e_{i} b_{i},
$$

where $e_{i}, i=1,2, \ldots, n$, are minimal idempotents, and $a_{i}, b_{i} \in \mathfrak{A}$. We then have

$$
\begin{aligned}
\mathfrak{A}=1 \cdot \mathfrak{A} \cdot 1 & =\sum_{i=1}^{n} \sum_{j=1}^{n} a_{i} e_{i} b_{i} \mathfrak{A} a_{j} e_{j} b_{j} \\
& =\sum_{i=1}^{n} \sum_{j=1}^{n} a_{i}\left(e_{i} \mathfrak{A} e_{j}\right) b_{j} .
\end{aligned}
$$

Since each space $e_{i} \mathfrak{A} e_{j}$ has dimension of at most one [1, Theorem 31.6], each subspace $a_{i}\left(e_{i} \mathfrak{A} e_{j}\right) b_{j}$ has dimension of at most one. It follows that $\mathfrak{A}$ is finite dimensional. This completes the proof. 
If every maximal ideal in $\mathfrak{A}$ is of finite codimension or every maximal left ideal of $\mathfrak{A}$ is complemented, then the condition of Theorem 5 holds automatically. Therefore Theorem 5 covers [6, Theorem 2.2] and [8, Proposition 2.3].

Recall that a simple algebra is an algebra which has no proper ideals other than the zero ideal. For this sort of Banach algebra we have the following.

Corollary 6. Suppose that $\mathfrak{A}$ is a contractible or a reflexive amenable Banach algebra. If $\mathfrak{A}$ is also a simple algebra and has a maximal left or right ideal which is complemented in $\mathfrak{A}$, then $\mathfrak{A}$ is of a finite dimension.

Proof: In this case, $\{0\}$ is the only maximal ideal and is contained in a maximal left or a maximal right ideal which is complemented in $\mathfrak{A}$ by the assumption.

COROLlary 7 . Suppose that $\mathfrak{A}$ is a contractible or a reflexive amenable Banach algebra. If every maximal ideal in $\mathfrak{A}$ is either a maximal left or a maximal right ideal, then $\mathfrak{A}$ has finite dimension.

Proof: For each maximal ideal $M, \mathfrak{A} / M$ is a simple Banach algebra. Since $M$ itself is a maximal left or maximal right ideal in $\mathfrak{A}, \mathfrak{A} / M$ has either no non-zero proper left ideals or no non-zero proper right ideals, meaning that $\{0\}$ is either a maximal left or a maximal right ideal in $\mathfrak{A} / M$ which is certainly complemented. From the preceding corollary, $\mathfrak{A} / M$ is of finite dimension. Then any subspace containing $M$ is finite codimensional and hence complemented in $\mathfrak{A}$. This is true for every maximal ideal $M$ and so, from Theorem $5, \mathfrak{A}$ is finite dimensional.

REMARK 8. If $\mathfrak{A}$ is commutative, then the condition of Corollary 7 is satisfied automatically. Therefore Corollary 7 covers [2, Theorem 6.2$]$.

\section{REFERENCES}

[1] F.F. Bonsall and J. Duncan, Complete Normed Algebras (Springer-Verlag, Berlin, Heidelberg, New York, 1973).

[2] P.C. Curtis, Jr. and R.J. Loy, 'The structure of amenable Banach algebras', J. London Math. Soc. 40 (1989), 89-104.

[3] J.E. Galé, T.J. Ransford and M.C. White, 'Weakly compact homomorphisms', Trans. Amer. Math. Soc. 331 (1992), 815-824.

[4] F. Ghahramani, R.J. Loy and G.A. Willis, 'Amenability and weak amenability of second conjugate Banach algebras', Proc. Amer. Math. Soc. 124 (1996), 1489-1497.

[5] A.Ya. Helemskii, Banach and locally convex algebras (Oxford University Press, Oxford, New York, Toronto, 1993).

[6] B.E. Johnson, 'Weakly compact homomorphisms between Banach algebras', Math. Proc. Cambridge Philos. Soc. 112 (1992), 157-163.

[7] B.E. Johnson, Cohomology in Banach algebras, Mem. Amer. Math. Soc. 127, 1972.

[8] V. Runde, 'The structure of contractible and amenable Banach algebras', in Banach Algebras '97 (Blaubeuren) (de Gruyter, Berlin, 1998), pp. 415-430. 
[9] J.L. Taylor, 'Homology and cohomology for topological algebras', Adv. Math. 9 (1972), 137-182.

Department of Mathematical Sciences

University of Alberta

Edmonton, Alberta

T6G 2G1, Canada

e-mail: yzhang@math.ualberta.ca 